\title{
Modeling and Numerical Analysis of Seismo-Electromagnetics Perturbation Using FDTD Method
}

\author{
Hendy Santosa ${ }^{1}$, Yasuhide Hobara ${ }^{2}$, and Achmad Munir $^{3}$ \\ ${ }^{1}$ Electrical Engineering Faculty, Bengkulu University \\ Jl. Raya Kandang Limun Bengkulu, Indonesia \\ ${ }^{2}$ Department of Communication Engineering and Informatics \\ Graduate School of Informatics and Engineering, The University of Electro-Communication \\ 1-5-1 Chofugaoka, Chofu, Tokyo 182-8585, Japan \\ ${ }^{3}$ Radio Telecommunication and Microwave Laboratory, \\ School of Electrical Engineering and Informatics \\ Institut Teknologi Bandung, Jalan Ganesha 10 Bandung 40132, Indonesia
}

\begin{abstract}
The modeling and numerical analysis of seismo-electromagnetics perturbation are approached using finite-difference time-domain (FDTD) method. The research related seismo-electromagnetics perturbation recently has been implemented for earthquakes (EQs) precursor analysis by utilizing very low frequency (VLF) wave propagation. Here, the proposed method in two-dimensional (2D) form is applied to compute electromagnetics fields of the observed VLF wave and to investigate the phenomena of seismo-electromagnetics perturbation by modeling the geographical maps in two dimensions. To truncate the region of computation, the boundary condition of split-field perfectly matched layer (PML) is implemented. The parameters of investigation including the change of amplitude, frequency and time-shifting in the VLF wave are interpreted in term of changing the ionosphere layer which is confirmed as seismo-electromagnetics perturbation. From numerical result, it shows that the approaching technique using FDTD method is successfully to model the correlation between VLF wave propagation and perturbation wave associated with earthquake for different location. Moreover, some results related to time-shifting of VLF wave and frequency-influence of perturbation wave are also presented.
\end{abstract}

Keywords: FDTD method, perturbation wave, seismo-electromagnetics, split-field $P M L$

\section{Introduction}

Since along time ago, there have been a wide variety of natural disasters in the world including hurricane, tsunami, volcano eruption, and earthquakes (EQs), which need more attentions for preventing actions. In accordance to these matters, Indonesia as one of the most disaster prone countries, has formulated the National Plan on Disaster Management 2010-2014 in which the disaster management could be conducted more being integrated, coordinated and comprehensive [1]. Of the many disasters that have occurred, earthquake is one of the most disasters which is frequently and recently happened such as Aceh EQ, Jogjakarta EQ, Tasikmalaya EQ, Papua EQ etc. that indicates how large the outspread of EQ and its hazard. In order to minimize the impact of the EQ disaster, the EQ precursor or prediction is becoming a primary important thing to be conducted.

In last decade, many theoretical analysis and experimental campaigns have been conducted to investigate electromagnetics waves phenomena related earthquakes [2]-[7]. One of key electromagnetics wave analyses is the utilization of VLF (Very Low Frequency) wave transient since the large change of vertical charge moment in VLF wave is one of crucial condition to generate perturbation effect which indicates the exciting condition of electromagnetic waves in the ionosphere. The change of charge moment can be derived remotely from VLF wave

Received: September $20^{\text {th }}, 2013$. Accepted: March $24^{\text {th }}, 2014$ 
measurement. The theoretical and numerical works have been carried out by numerous authors to analyze the scattering process from the VLF wave propagation under the existence of ionospheric perturbation [4]-[10]. Small attention has been paid to quantitatively compare the observed amplitude and time shifting values of the simulation result [10]. This kind of approach is quite important in deriving the quantitative characteristic of scattering source as well as the characteristics of VLF waves.

The conventional EQ prediction is usually conducted based on the movement of crustal earth [3]; however this kind of mechanical measurement has been concluded to be not so useful for analyzing short-term EQs predictions. In recent times, wave measurements by means of electromagnetic effects have been one of the effective methods for EQs prediction [3]-[5]. This method can principally be classified into two categories; the first is the detection of radio emissions from the hypocenter, and the second is to detect an indirect effect of EQs taking place in the atmosphere and ionosphere by means of the pre-exciting radio transmitter signals. In this paper, the approach to model electromagnetics wave perturbation related earthquakes and to compute electromagnetics field of the observed VLF waves are proposed by use of finitedifference time-domain (FDTD) method. The scattering amplitude from the observed VLF waves of observation points is analyzed to confirm the electromagnetics wave perturbation. The analysis of scattered amplitude for expected distance variation from the observation point is also included in the discussion.

\section{FDTD and Boundary Condition}

\section{A. FDTD Method}

With the continued growth of computing power, the modeling and numerical simulation have grown immensely as a tool for understanding and analyzing any problem in science. In electromagnetic problems, there are quite a number of useful methods including the Method of Moments, Finite Volume methods, Finite Element methods, and Spectral methods, just to name a few. Finite-Difference Time-Domain (FDTD) method, however, grew to become the method of choice in the 1990s, for a number of reasons [11]-[12]. It has always the advantage of being a very simple method that the derivation of difference equations is straightforward. The method is also extremely useful when a transient or broadband analysis is required such as for analyzing the scattering pattern of a broadband pulse. However, in cases where the steady state solution is sought only at a single frequency, the method is rather inefficient. Instead, frequency domain methods can be more efficient, since they avoid the need to step in time.

To model the electromagnetics wave phenomena as well as seismo-electromagnetics perturbation using FDTD method, the following Maxwell's equations are applied for the formulation.

$$
\begin{aligned}
& \frac{\partial \mathbf{H}}{\partial \boldsymbol{t}}=\frac{1}{\varepsilon} \nabla \times \mathbf{E} \\
& \frac{\partial \mathbf{E}}{\partial \boldsymbol{t}}=-\frac{1}{\mu} \nabla \times \mathbf{H}
\end{aligned}
$$

where $\mathbf{E}$ and $\mathbf{H}$ are the electric field and magnetic field vectors, respectively, whilst $\varepsilon$ and $\mu$ are the permittivity and the permeability, respectively.

Based on the FDTD notation [11]-[12], the placement of electric field and magnetic field components for two-dimensionally (2D) transverse electric (TE) wave mode is allocated. The small vectors with thick arrows are placed at the point in the mesh at which they are defined and stored. A portion of Yee cell constituting a unit cell for the TE wave mode is depicted in Figure 1. It is seen that the magnetic field $(H)$ component is located between the spatial positions of the two electric field $(E)$ components. The $E_{x}$ component is located at half $x$ and integer $y$ grid points, i.e. $(i+1 / 2, j)$, while the $E_{y}$ component is located at integer $x$ and half $y$ grid points, i.e. $(i$, $j+1 / 2)$. The magnetic field component $H_{z}$ is located at half $x$ and half $y$ grid points, i.e. $(i+1 / 2$, 
$j+1 / 2)$. Then by using the FDTD notation, the electric and magnetic field components for $2 \mathrm{D}$ TE wave mode are expressed as follows.

$$
\begin{aligned}
& \left.E_{x}\right|_{i+\frac{1}{2}, j} ^{n+1}=\left.E_{x}\right|_{i+\frac{1}{2}, j} ^{n}+\frac{\Delta t}{\varepsilon_{i+\frac{1}{2}, j} \Delta y}\left[\left.H_{z}\right|_{i+\frac{1}{2}, j+\frac{1}{2}} ^{n+\frac{1}{2}}-\left.H_{z}\right|_{i+\frac{1}{2}, j-\frac{1}{2}} ^{n+\frac{1}{2}}\right] \\
& \left.E_{y}\right|_{i, j+\frac{1}{2}} ^{n+1}=\left.E_{y}\right|_{i, j+\frac{1}{2}} ^{n}-\frac{\Delta t}{\varepsilon{ }_{i+\frac{1}{2}, j} \Delta x}\left[\left.H_{z}\right|_{i+\frac{1}{2}, j+\frac{1}{2}} ^{n+\frac{1}{2}}-\left.H_{z}\right|_{i-\frac{1}{2}, j+\frac{1}{2}} ^{n+\frac{1}{2}}\right] \\
& \left.H_{y}\right|_{i+\frac{1}{2}, j+\frac{1}{2}} ^{n+\frac{1}{2}}=\left.H_{z}\right|_{i+\frac{1}{2}, j+\frac{1}{2}} ^{n-\frac{1}{2}}+\frac{\Delta t}{\mu t+\frac{1}{2}, j+\frac{1}{2}}\left[\frac{\left.E_{x}\right|_{i+\frac{1}{2}, j+1} ^{n}-\left.E_{x}\right|_{i+\frac{1}{2}, j} ^{n}}{\Delta y} \frac{\left.E_{y}\right|_{i+1, j+\frac{1}{2}} ^{n}-\left.E_{y}\right|_{i, j+\frac{1}{2}} ^{n}}{\Delta x}\right]
\end{aligned}
$$

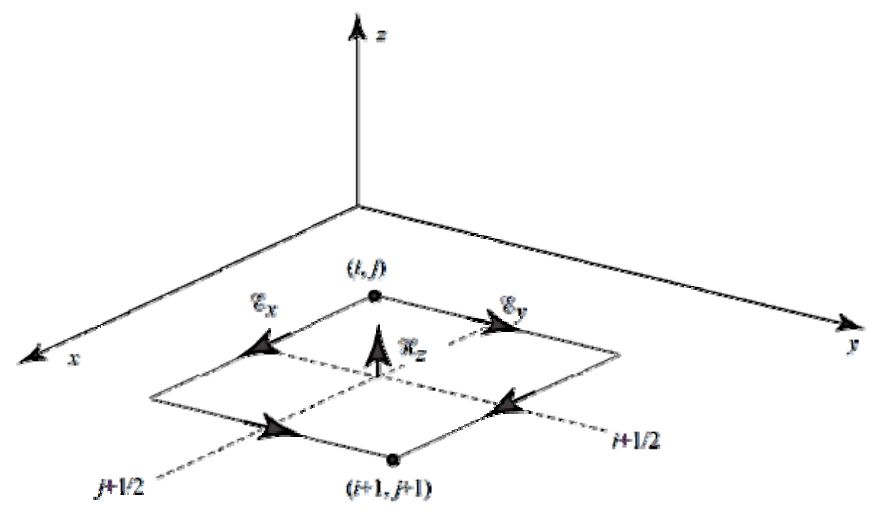

Figure 1. A unit cell for 2D TE wave mode for FDTD method

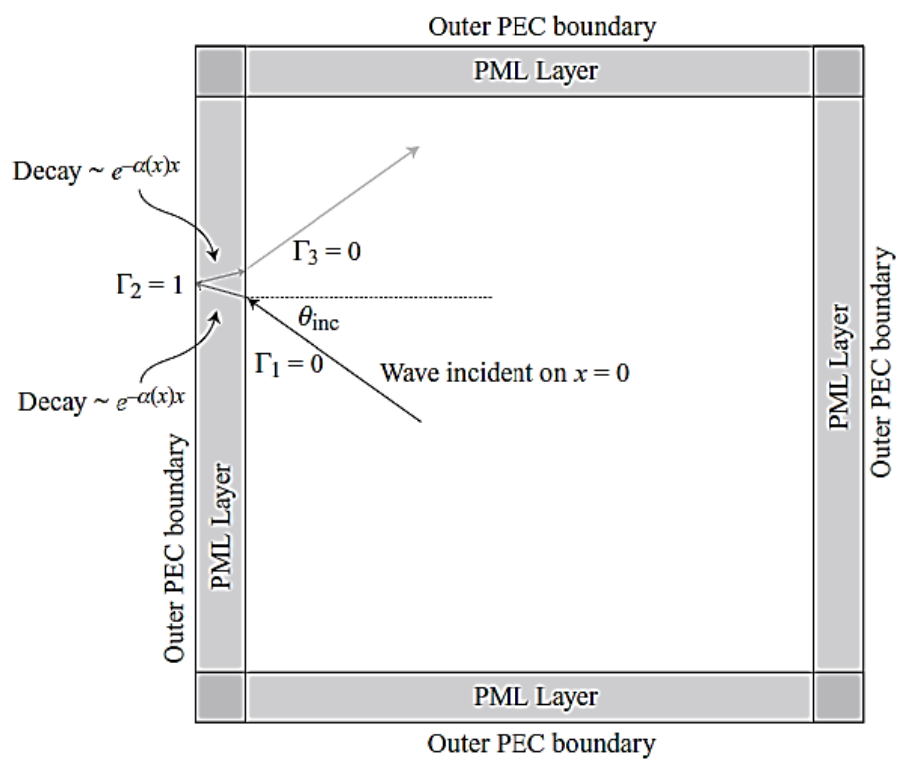

Figure 2. Perfectly matched layer (PML) boundary condition applied for computation 
Hendy Santosa, et al.

\section{B. Split-Field PML}

As shown in Figure 2, the perfectly matched layer (PML) method involves modifying the medium of simulation in a thin layer around the boundary, so that the layer becomes an artificially absorbing or lossy medium. The boundary layer which is applied to truncate the region of computation is designed so that it absorbs enough of the outgoing wave so that reflections from the actual boundary are acceptably low. The modification of medium is conducted by introducing the conductivity $(\sigma)$ in boundary layers. In addition, however, the boundary layer should be designed to prevent reflections from the interface between the actual medium and the boundary medium, i.e. $\Gamma_{1}=0$. This means that the two media should have an impedance-matched to obtain a very high accuracy of computation. Here, the Berenger's method known as split-field PML method is applied for the computation as it works based on field-splitting of Maxwell's equations and selectively choosing different values for the conductivities $\left(\sigma_{e}\right.$ and $\left.\sigma_{m}\right)$ in different directions.

The following equations are showing the expression of Maxwell's equations for the TE wave mode where $\sigma_{e} \neq 0$ and $\sigma_{m} \neq 0$ applied for split-field PML method.

$$
\begin{aligned}
& \varepsilon_{2} \frac{\partial \boldsymbol{E}_{\boldsymbol{X}}}{\partial \boldsymbol{t}}+\sigma_{\boldsymbol{e}, \boldsymbol{y}} \boldsymbol{E}_{\boldsymbol{x}}=\frac{\partial \boldsymbol{H}_{\boldsymbol{z}}}{\partial \boldsymbol{y}} \\
& \varepsilon_{2} \frac{\partial \boldsymbol{E}_{\boldsymbol{y}}}{\partial \boldsymbol{t}}+\sigma_{\boldsymbol{e}, \boldsymbol{x}} \boldsymbol{E}_{\boldsymbol{y}}=\frac{\partial \boldsymbol{H}_{\boldsymbol{z}}}{\partial \boldsymbol{x}} \\
& \mu_{2} \frac{\partial \boldsymbol{H}_{\boldsymbol{z} \boldsymbol{x}}}{\partial \boldsymbol{t}}+\sigma_{\boldsymbol{m}, \boldsymbol{x}} \boldsymbol{H}_{\boldsymbol{z} \boldsymbol{x}}=-\frac{\partial \boldsymbol{E}_{\boldsymbol{y}}}{\partial \boldsymbol{x}} \\
& \mu_{2} \frac{\partial \boldsymbol{H}_{\boldsymbol{z}}}{\partial \boldsymbol{t}}+\sigma_{\boldsymbol{m}, \boldsymbol{y}} \boldsymbol{H}_{\boldsymbol{z} \boldsymbol{y}}=-\frac{\partial \boldsymbol{E}_{\boldsymbol{x}}}{\partial \boldsymbol{y}}
\end{aligned}
$$

The equations will modify the regular Maxwell's equations, i.e. (1) and (2), for $\sigma_{e, x}=\sigma_{e, y}=$ $\sigma_{e}$ and $\sigma_{m, x}=\sigma_{m, y}=\sigma_{m}$. The separation of conductivities into two parts provides the possibility to selectively attenuate the fields in one direction. For instance, if $\sigma_{e, x}=\sigma_{m, x}=0$ while $\sigma_{e, y} \neq 0$ and $\sigma_{m, y} \neq 0$, then the waves with field components of $E_{x}$ and $H_{z y}$ which propagate along the $y$ direction are absorbed, whilst the waves with field components of $E_{y}$ and $H_{z x}$ which propagate along the $x$-direction are transmitted. At this point, the PML properties are started to work. In case the nonzero conductivity pairs $\sigma_{e, y}$ and $\sigma_{m, y}$ at the interface of region-1/region-2 perpendicular to the $y$-direction are applied, then the components of waves which propagate only in the $y$-direction are attenuated.

The incident wave in Figure 2 will enter the PML layer without reflection, but it then will be attenuated at rate of $\alpha_{P M L}($ Neper $/ \mathrm{m})$ over the thickness of the PML, here is denoted by $\Delta_{P M L}$. Upon reaching the end of the PML layer, the waves will reflect perfectly from the PEC boundary and continue to attenuate with the same rate of $\alpha_{P M L}$ over the thickness of the PML again, at which point it will re-enter the simulation space. In essence the distance of propagated will depend on incident angle, and the rates of attenuation depend on angle and frequency. Furthermore, since the electric field $(E)$ and the magnetic field $(H)$ are interleaved by half a spatial step, they will encounter the PML boundary differently. To reduce the resulting reflection error, therefore it uses grading factor for the PML conductivity smoothly from zero to some maximum value at the outer boundary [12]. For most FDTD simulation, the PML layer is typically taken to be about 10 cells thick [11]-[12].

Furthermore, to implement the FDTD system for a region having a continuous variation of material properties with spatial position, it is desirable to define and store the following constant updating coefficient for each field component before the time stepping begin [13]. For electric field components, the updating coefficients at point $(i, j)$ are: 


$$
\begin{aligned}
& \left.C_{a}\right|_{i, j}=\left(1-\frac{\sigma_{i, j} \Delta t}{2 \varepsilon_{i, j}}\right) /\left(1+\frac{\sigma_{i, j} \Delta t}{2 \varepsilon_{i, j}}\right) \\
& \left.C_{b 1}\right|_{i . j}=\left(\frac{\Delta t}{\varepsilon_{i, j} \Delta_{1}}\right) /\left(1+\frac{\sigma_{i, j} \Delta t}{2 \varepsilon_{i, j}}\right) \\
& \left.C_{b 2}\right|_{i, j}=\left(\frac{\Delta t}{\varepsilon_{i, j} \Delta_{2}}\right) /\left(1+\frac{\sigma_{i, j} \Delta t}{2 \varepsilon_{i, j}}\right)
\end{aligned}
$$

Whilst for magnetic field components, the updating coefficient at point $(i, j)$ are:

$$
\begin{aligned}
& \left.\boldsymbol{D}_{a}\right|_{i, j}=\left(1-\frac{\sigma_{i, j}^{*} \Delta t}{2 \mu_{i, j}}\right) /\left(1+\frac{\sigma_{i, j}^{*} \Delta t}{2 \mu_{i, j}}\right) \\
& \left.\boldsymbol{D}_{b 1}\right|_{i, j}=\left(\frac{\Delta t}{\mu_{i, j} \Delta_{1}}\right) /\left(1+\frac{\sigma_{i, j}^{*} \Delta t}{2 \mu_{i, j}}\right) \\
& \left.\boldsymbol{D}_{b 2}\right|_{i . j}=\left(\frac{\Delta t}{\varepsilon_{i, j} \Delta_{2}}\right) /\left(1+\frac{\sigma_{i, j}^{*} \Delta t}{2 \mu_{i, j}}\right)
\end{aligned}
$$

For a square lattice of cell, $\Delta x=\Delta y=\Delta$, and thus $\Delta_{1}=\Delta_{2}=\Delta$. For this case then $C_{b 1}=C_{b 2}$ and $D_{b 1}=D_{b 2}$, so it can reduce the storage requirement to two updating coefficients per field components. Therefore, the 2D TE wave mode FDTD notation for the electric and magnetic field components with media which have different permittivity and conductivity are expressed as follows.

$$
\begin{aligned}
& \boldsymbol{u}=\left.\left.\operatorname{media} E_{\boldsymbol{X}}\right|_{i+\frac{1}{2}, j} \Rightarrow E_{X}\right|_{i+\frac{1}{2}, j} ^{n+1}=\left.C_{a}(u) E_{x}\right|_{i+\frac{1}{2}, j} ^{n}+C_{b}(u)\left[\left.H_{z}\right|_{i+\frac{1}{2}, j+\frac{1}{2}} ^{n+\frac{1}{2}}-\left.H_{z}\right|_{i+\frac{1}{2}, j-\frac{1}{2}} ^{n+\frac{1}{2}}\right] \\
& v=\left.\left.\operatorname{media} E_{y}\right|_{i, j+\frac{1}{2}} \Rightarrow E_{y}\right|_{i, j+\frac{1}{2}} ^{n+1}=\left.C_{a}(v) E_{y}\right|_{i, j+\frac{1}{2}} ^{n}-C_{b}(v)\left[\left.H_{z}\right|_{i+\frac{1}{2}, j+\frac{1}{2}} ^{n+\frac{1}{2}}-\left.H_{z}\right|_{i-\frac{1}{2}, j+\frac{1}{2}} ^{n+\frac{1}{2}}\right] \\
& \boldsymbol{w}=\text { media }\left.\boldsymbol{H}_{\boldsymbol{z}}\right|_{\boldsymbol{i}+\frac{1}{2}, j+\frac{1}{2}} \Rightarrow \\
& \left.H_{z}\right|_{i+\frac{1}{2}, j+\frac{1}{2}} ^{n+\frac{1}{2}}=\left.D_{a}(w) H_{z}\right|_{i+\frac{1}{2}, j+\frac{1}{2}} ^{n-\frac{1}{2}}+D_{b}(w)\left[\frac{\left.E_{x}\right|_{i+\frac{1}{2}, j+1} ^{n}-\left.E_{x}\right|_{i+\frac{1}{2}, j} ^{n}}{\Delta y}-\frac{\left.E_{y}\right|_{i+1, j+\frac{1}{2}} ^{n}-\left.E_{y}\right|_{i, j+\frac{1}{2}} ^{n}}{\Delta x}\right]
\end{aligned}
$$

\section{Modeling, Numerical Analysis and Discussion}

\section{A. Seismo-electromagnetics Modeling}

To solve Maxwell's equations for the seismo-electromagnetics modeling, the FDTD method is directly applied by separating the analysis region into the numerous small finite square lattice cells. The two-dimensionally (2D) FDTD method is used for computational simplicity since the method requires substantial computer memories and CPU resources for the increase of number of Yee cells. The geometry of geographical maps for modeling is depicted in Figure 3 (read line shaded area), whereas the simulation parameter for modeling is summarized in Table 1. To mimic the real condition of modeling, the permittivity of sea-water $\left(\varepsilon_{1}\right)$ and land $\left(\varepsilon_{2}\right)$ is set to be 7.0 and 4.0 , respectively. Hence, the conductivity of sea-water $\left(\sigma_{1}\right)$ 
and land $\left(\sigma_{2}\right)$ is assumed to be $4.0 \times 10^{-7} \mathrm{~S} / \mathrm{m}$ and $3.0 \times 10^{-7} \mathrm{~S} / \mathrm{m}$, respectively. The continuous sine-wave with frequency of $18.2 \mathrm{kHz}$ is used to simulate the VLF transmitter in India and its receiver in Indonesia with the distance of about $3,774 \mathrm{~km}$. In the simulation, the model of EQs to analyze the seismo-electromagnetics perturbation is represented by Gaussian pulse wave. The square cells with each cell size of $1.648 \mathrm{~km}$ are dividing the simulation area into 2,290 cells and 432 cells for length and width area, respectively. The total number of cells for the simulation is therefore 989,280 cells. It is note that the cell size is one-tenth of wavelength used for the simulation. The split-filed PML boundary layers about 10 cells thick are implemented surrounding the area of simulation to truncate the region of computation.

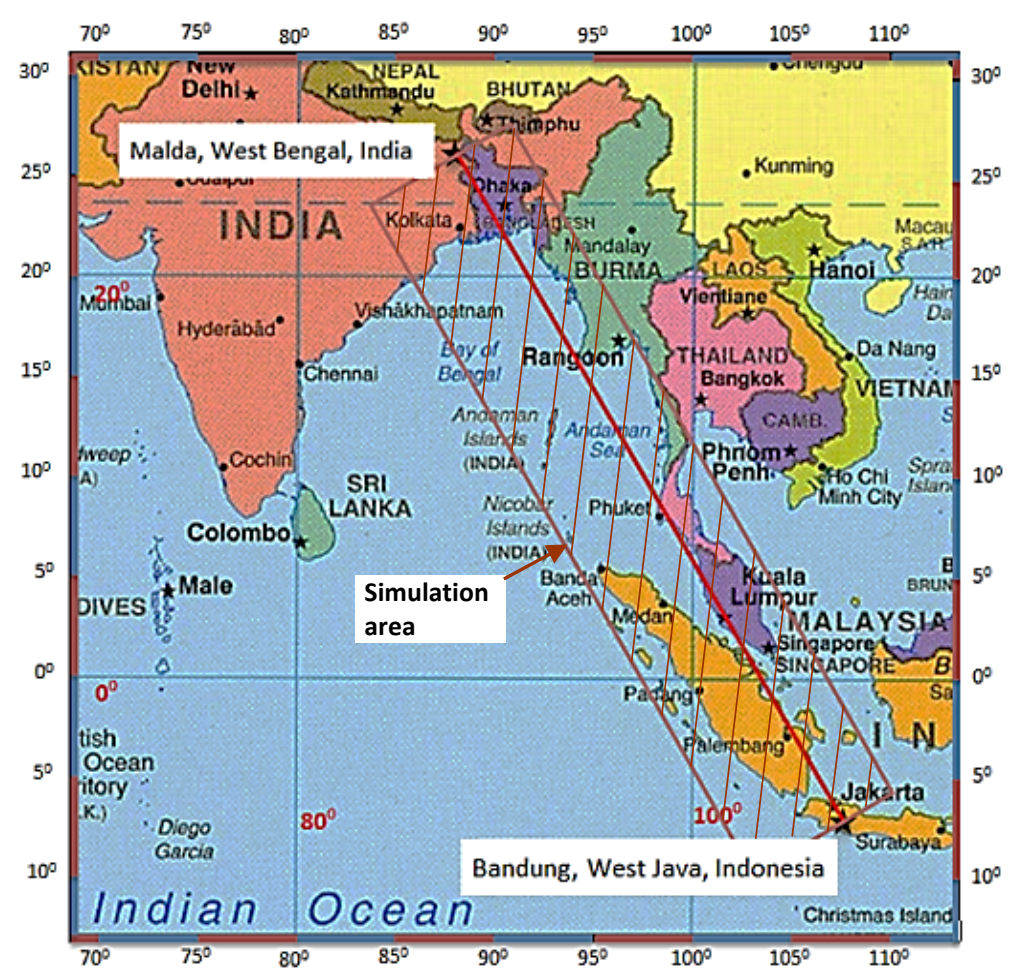

Figure 3. Geometry of geographical maps for modeling which consists of VLF transmitter in India and its receiver in Indonesia

Table 1. Simulation parameter for 2D FDTD modeling

\begin{tabular}{|c|l|l|}
\hline No. & Parameter & Value \\
\hline 1 & Sine wave frequency & $18.2 \mathrm{kHz}$ \\
\hline 2 & Length of simulation area & $3,774 \mathrm{~km}$ \\
\hline 3 & Width of simulation area & $712 \mathrm{~km}$ \\
\hline 4 & Sea-water permittivity $\left(\varepsilon_{1}\right)$ & 7.0 \\
\hline 5 & Land permittivity $\left(\varepsilon_{2}\right)$ & 4.0 \\
\hline 6 & Sea-water conductivity $\left(\sigma_{1}\right)$ & $4.0 \times 10^{-7} \mathrm{~S} / \mathrm{m}$ \\
\hline 7 & Land conductivity $\left(\sigma_{2}\right)$ & $3.0 \times 10^{-9} \mathrm{~S} / \mathrm{m}$ \\
\hline
\end{tabular}

\section{B. Numerical Analysis and Discussion}

The flow chart of numerical analysis for seismo-electromagnetics modeling with perturbation wave is shown in Figure 4, where the modeling is performed using 2D FDTD with TE wave mode and simulated with Matlab®. 


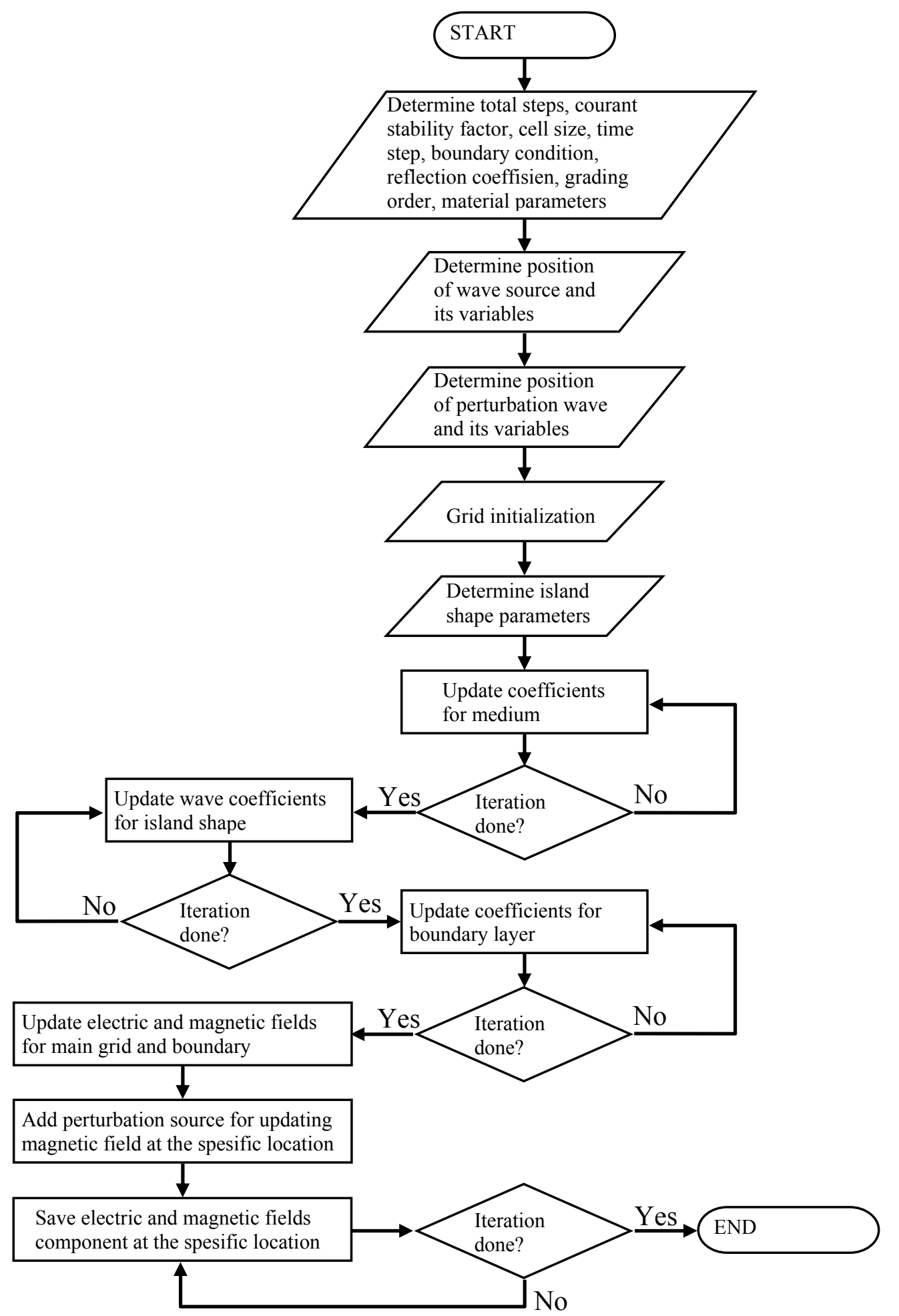

Figure 4. Flow chart of seismo-electromagnetics modeling with perturbation wave using $2 \mathrm{D}$ FDTD TE wave mode 
The simulation result of electromagnetics waves field distribution is shown in Figure 5 which is captured at $68.681 \mathrm{~ms}$ after the continuous sine-wave of VLF transmitter is generated. It shows that amplitude of electromagnetics waves varies when propagating in the different medium. This is indicated by different colors of simulation result. The propagation path is selected from the VLF transmitter in India to the receiver in Indonesia with the distance of about $3,774 \mathrm{~km}$. The local transient electromagnetic waves in the ionospheric layer are considered due to the perturbation distance. The orange star represents observation point is located at $3316.480 \mathrm{~km}$ in the $x$-direction and $623.076 \mathrm{~km}$ in the $y$-direction. The purple square represents perturbation point which is located at $2307.69 \mathrm{~km}$ in the $x$-direction and $329.67 \mathrm{~km}$ in the $y$ direction. Furthermore, the red triangle represents perturbation point which is located at $2967.03 \mathrm{~km}$ in the $x$-direction and $494.505 \mathrm{~km}$ in the $y$-direction.

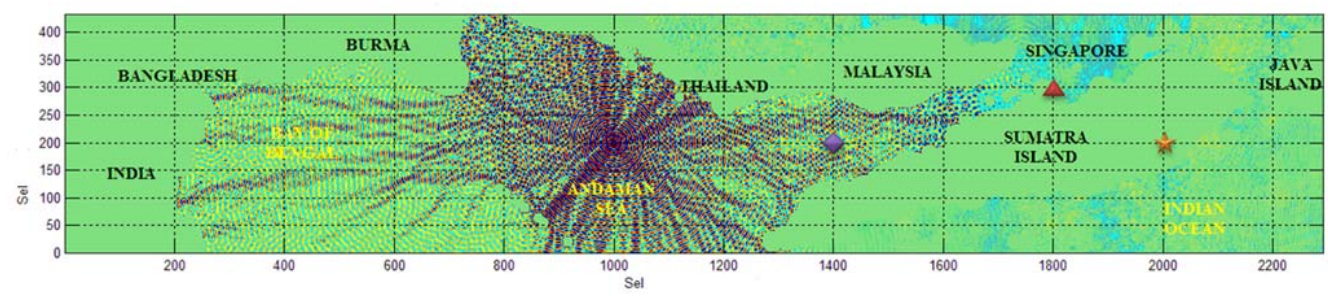

Figure 5. Simulated result of electromagnetics waves field distribution

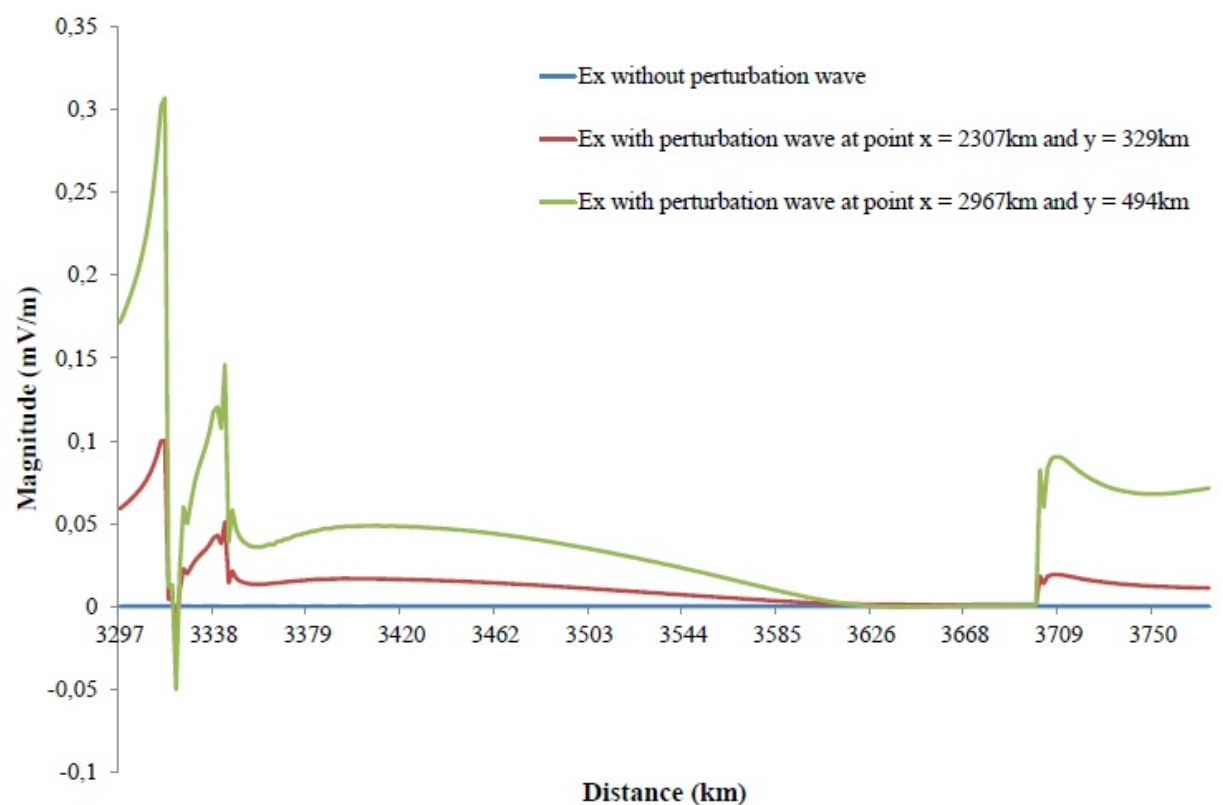

Figure 6. Simulated result of electric field amplitude at $x$-direction without and with perturbation in the different location.

Figure 6 depicts the simulated results for temporal dependences of amplitude in which the electric field is observed between $3,297 \mathrm{~km}$ to $3,774 \mathrm{~km}$ in $x$-direction and at $623 \mathrm{~km}$ in $y$ direction. As plotted in the figure, the blue line represents continuous sine-wave without perturbation, whereas the red and green lines represent the sine-waves with perturbation. The observation point for the red line is located at $2,307 \mathrm{~km}$ in $x$-direction and $329 \mathrm{~km}$ in $y$-direction, whilst the green line is at $2,967 \mathrm{~km}$ in $x$-direction and $494 \mathrm{~km}$ in $y$-direction. From the figure, it should be noted that considerable changes in amplitude of VLF wave are obtained. The change is characterized by the distance between the point of perturbation wave and the location of 
observation which the green line has the nearest distance from the observation. The amplitude difference of electric fields between the perturbation waves is $207.223 \mathrm{mV} / \mathrm{m}$.

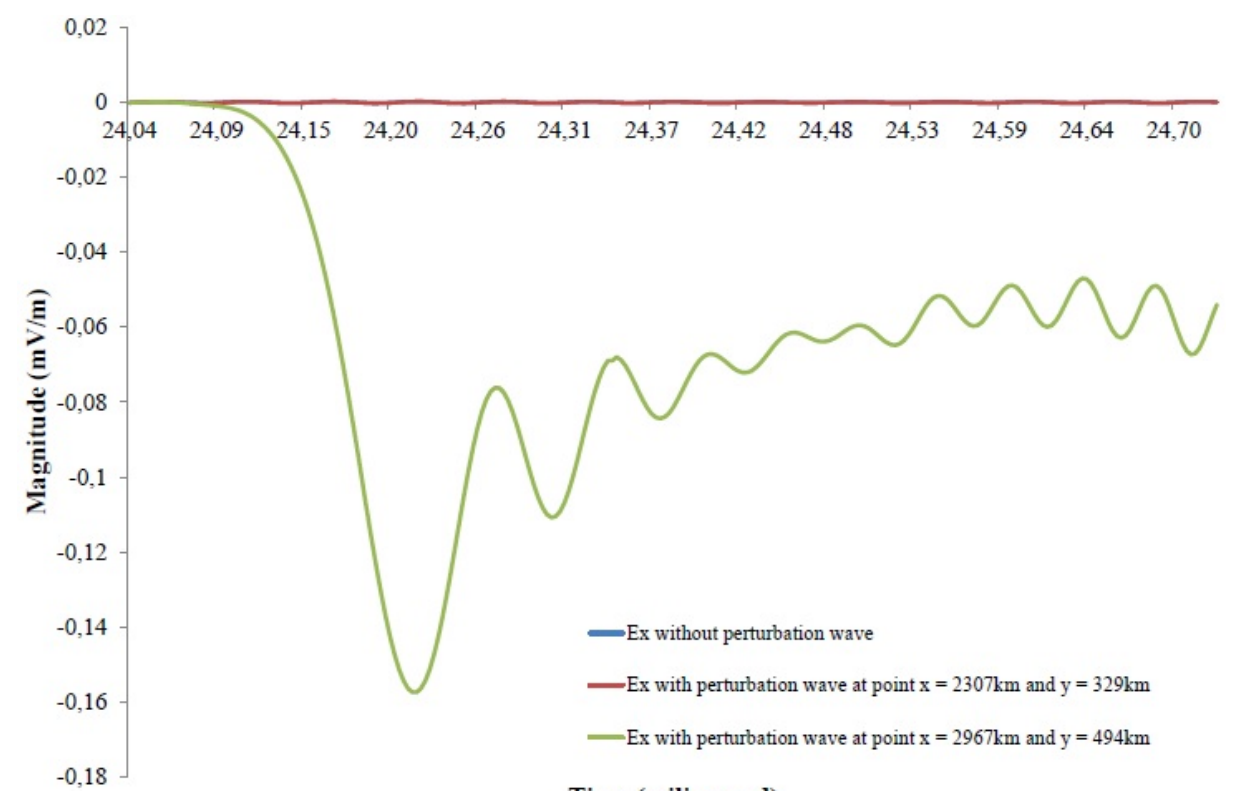

Time (milisecond)

Figure 7. Simulated result of electric field time shifting at $x$-direction with and without perturbation in the different location at time $24.038 \mathrm{~ms}$ to $24.698 \mathrm{~ms}$

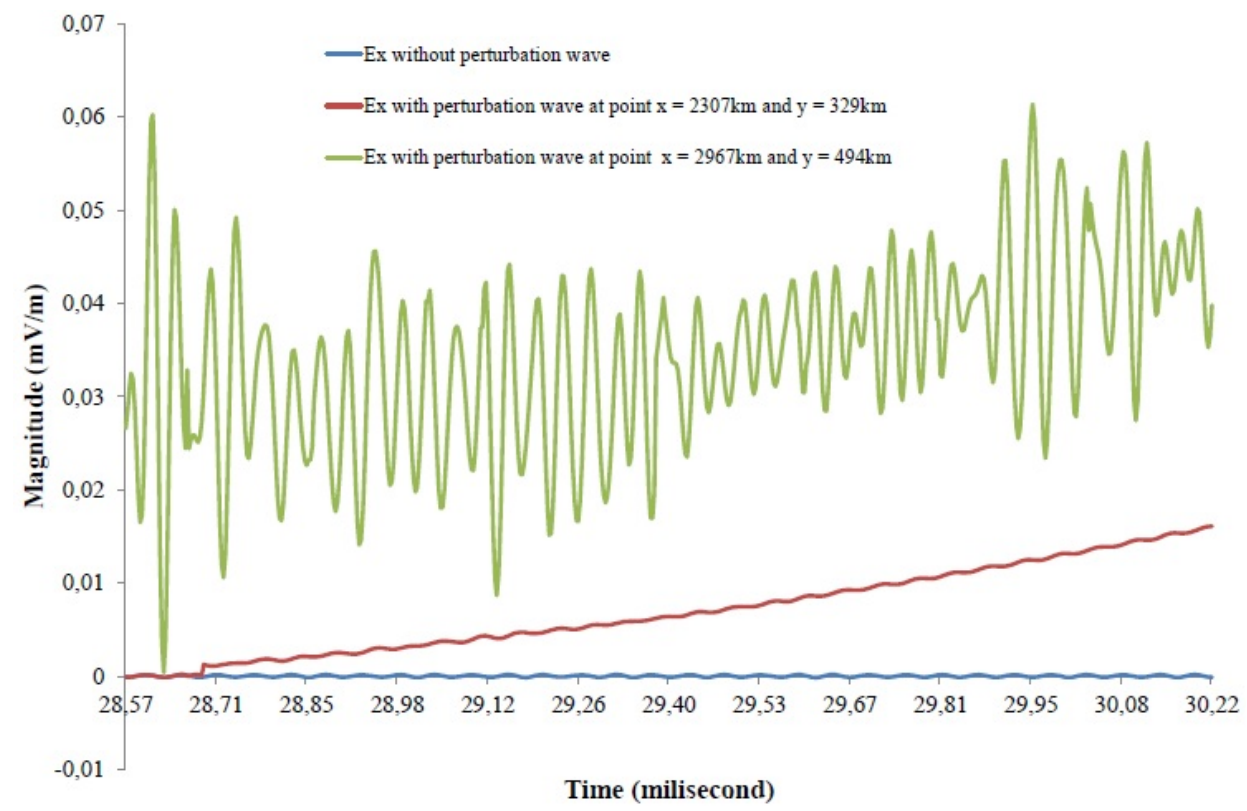

Figure 8. Simulated result of electric field time shifting at $x$-direction with and without perturbation in the different location at time $29.533 \mathrm{~ms}$ to $30.192 \mathrm{~ms}$

Moreover, Figures 7 and 8 plot the delay time of the scattered amplitude at $t=5.445 \mathrm{~ms}$ for VLF wave without and with perturbation wave at $x$-direction of $2,307 \mathrm{~km}$ and $y$-direction of $329 \mathrm{~km}$ (the blue line), and $x$-direction of $2,967 \mathrm{~km}$ and $y$-direction of $494 \mathrm{~km}$, respectively (the red and green lines). The results show that the different location of perturbation wave associated 
with earthquake influences to the speed of propagation wave to reach the location of observation. The closer perturbation wave location evokes the smaller time to reach the observation location. It notes that the speed of propagation wave is highly affected by the permittivity of medium where the speed in sea-water is slower than in the land. Hence from Figure 9, it is seen that Gaussian wave perturbation has no influence to the observation frequency but it gives the influence only to the amplitude of electromagnetic waves.

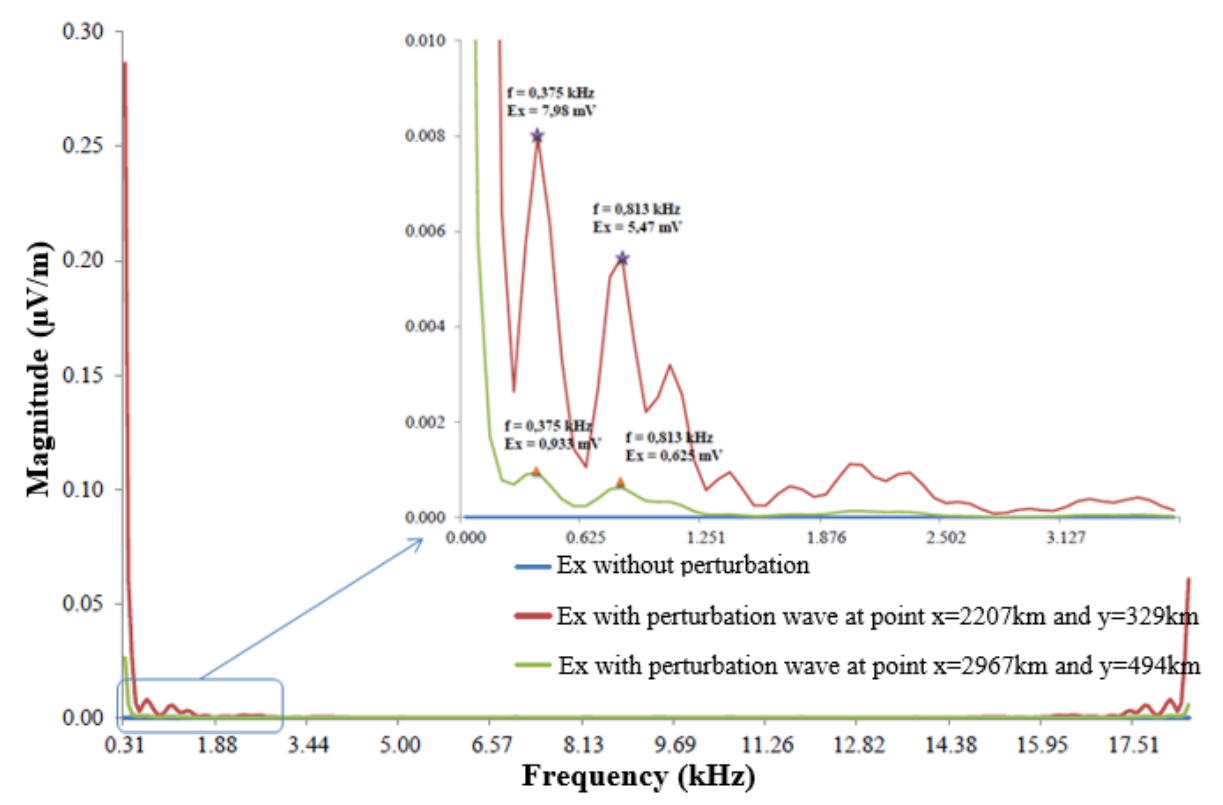

Figure 9. Simulated result of electric field frequency at $x$-direction with and without perturbation in the different location

However in the current simulation there are some problems to be overcome. As the simulation is performed in 2D, so the transmitter-receiver paths have to be selected in order the perturbation locations rather close. To study the wave scattering from the arbitrary perturbation location, the forthcoming numerical analysis in three-dimension (3D) form of FDTD method will be investigated.

\section{Conclusion}

The approach to model seismo-electromagnetics perturbation for earthquake precursor analysis around Indonesia and to analysis electromagnetics field of the observed VLF waves have been demonstrated by using 2D-FDTD method in the TE wave mode. The numerical analysis of scattering VLF waves which propagates with perturbation wave has been performed and the difference amplitude as well as time shifting has also been calculated. From the result, it has been confirmed that: (i) the amplitude of electromagnetics wave depends on the distance between the source of perturbation wave and the location of observation, (ii) electromagnetics wave perturbation of Gaussian has influenced to the amplitude of the VLF waves, instead of its frequency. In addition, a further investigation to model seismo-electromagnetics perturbation in three-dimension (3D) FDTD method is still in progress where the results will be reported later.

\section{Acknowledgements}

This work is partially supported by the Research Grant from Institut Teknologi Bandung (ITB) under the program scheme of Research and Innovation 2013 contract number DIPA023.04.2.189730/2013. 


\section{References}

[1] BNBP, National Disaster Management Plan 2010-2014, Indonesia, 2010.

[2] R. Barr, D. Llanwyn Jones, and C.J. Rodger, "ELF and VLF radio waves," Journal of Atmospheric and Solar-Terrestrial Physics, Vol. 62, Issue 17-18, pp. 1689-1718, Nov. 2000.

[3] M. Hayakawa, "Electromagnetic phenomena associated with earthquakes," Transworld Research Network, Trivandrum, 2009.

[4] Y. Hobara, M. Hayakawa, H. Fujii and K. Ohta, "VLF subionospheric disturbances and ELF transients associated with TLEs: observations and modeling," $1^{\text {st }}$ International Conference on Science with Very Low Frequency Radio Waves: Theory and Observation, AIP Conf. Proc., pp. 3-41, Kolkata, India, Mar. 2010.

[5] M. Hayawaka, "The use of subionospheric VLF/LF propagation for the study of lower ionospheric perturbation associated with earthquake," $1^{\text {st }}$ International Conference on Science with Very Low Frequency Radio Waves: Theory and Observation, AIP Conf. Proc., pp. 3-41, Kolkata, India, Mar. 2010.

[6] J. Kenneth and W. Lynn, "VLF waveguide propagation: The basics," $1^{\text {st }}$ International Conference on Science with Very Low Frequency Radio Waves: Theory and Observation, AIP Conf. Proc., pp. 3-41, Kolkata, India, Mar. 2010.

[7] I.G. Zakharov and O.F. Tyrnov, "Electromagnetic precursors of global seismic activity in the VLF range" $21^{\text {st }}$ International Crimean Conference Microwave and Telecommunication Technology (CriMiCo) 2011 Proc., pp. 1073-1074, Sevastopol, Ukraine, Sep. 2011.

[8] M. Thevenot, J.P. Berenger, T. Monediere, and F. Jecko, "A FDTD scheme for the computation of VLF-LF propagation in the anisotropic earth-ionosphere waveguide," Annales Des Télécommunications, Vol. 54, Issue 5-6, pp. 297-310, May-Jun. 1999.

[9] Y. Wang and Q. Cao, "Analysis of seismic electromagnetic phenomena using the FDTD method," IEEE Trans. on Antennas and Propagat., Vol. 59, Issue 11, pp. 4171-4180, Nov. 2011.

[10] H. Santosa and A. Munir, "Finite-difference time-domain method for seismoelectromagnetics modelling," $2^{\text {nd }}$ Asia-Pacific Pacific Conference on Antennas and Propagation (APCAP) 2013 Proc., Chiang Mai, Thailand, Aug. 2013.

[11] A. Taflove, "Computational electrodynamics, The finite-difference time-domain method," Artech House, 1995.

[12] U.S. Inan and R.A. Marshall, "Numerical electromagnetics, The FDTD method," Cambridge University Press, UK, 2011.

[13] W.H. Weedon and C.M. Rappaport, "A general method for FDTD modeling of wave propagation in arbitrary frequency-dispersive media," IEEE Trans. on Antennas and Propagat., Vol. 45, Issue 3, pp. 401-401, Mar. 1997.

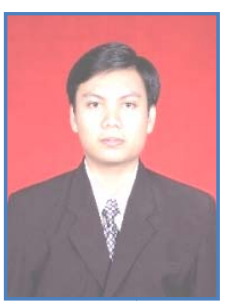

Hendy Santosa received the B.E. degree in Electrical Engineering from Gadjah Mada University, Indonesia, in 2004, the M.E. degree in Electrical Engineering (Telecommunication Engineering Option) from Institut Teknologi Bandung, Indonesia, in 2013. He is currently a lecturer with the Electrical Engineering Department, Faculty of Engineering at Bengkulu University, Indonesia. His research interests include electromagnetics wave propagation and its numerical analysis. 


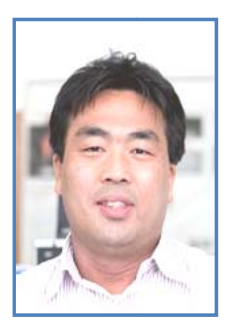

Yasuhide Hobara was born in Okayama, Japan on January 31, 1968. He received his M.E. and Ph.D. degrees in electrical engineering from the University of Electro-Communications, Japan in 1994 and 1997, respectively. He worked in various educational and research institutions as a Research Associate such as the University of Sheffield, UK, the Swedish Institute of Space Physics, Kiruna, Sweden, and LPCE/CNRS, France. Presently, he is a Professor in the Department of Electronic Engineering, the University of Electro-Communications in Tokyo, Japan. His research interests are on space plasma physics (plasma waves in the terrestrial and planetary environments), atmospheric electricity (electromagnetics phenomena from lightning, sprites and elves) and seismoelectromagnetics phenomena (electromagnetics waves and ionospheric perturbations). $\mathrm{He}$ is member of AGU and SAEJ.

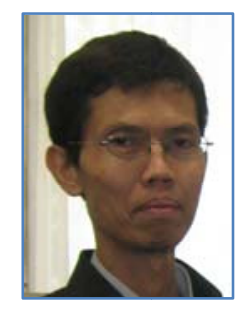

Achmad Munir received the B.E. degree in Electrical Engineering from Institut Teknologi Bandung, Indonesia, in 1995, the M.E. and D.E. degree in science and engineering from Yamaguchi University, Japan, in 2002 and 2005 respectively. From 2005 to 2007, he was a Research Fellow under JSPS fellowship program with department of Electrical and Electronics Engineering, Faculty of Engineering, Yamaguchi University, Japan, working on the artificial materials research, particularly, artificial dielectric and artificial magnetic materials. From 2007 to 2009, he was a Research Fellow with the Institute of Electronics, Communications, and Information Technology, Queens University Belfast, Northern Ireland, United Kingdom, involved in the experimental study of novel nonlinear artificial material including high impedance surface and artificial magnetic conductor for advanced EM applications. In January 2009, he joined the School of Electrical Engineering and Informatics, Institut Teknologi Bandung, Indonesia, as Lecturer. His research interests include linear and nonlinear artificial materials, electromagnetics wave propagation, and microwave devices. 